\title{
One size doesn't fit all: Supervised machine learning classification in athlete- monitoring
}

\author{
Matthew T.O. Worsey ${ }^{1,2}$, Hugo G. Espinosa. ${ }^{1 *}$, Jonathan B. Shepherd ${ }^{1}$ and David V. Thiel ${ }^{1 *}$ \\ ${ }^{1}$ Griffith University Sports Technology (GUST), School of Engineering and Built Environment, Griffith University, Brisbane, QLD 4111, \\ Australia. \\ ${ }^{2}$ Queensland Academy of Sport, Sport Performance Innovation and Knowledge Excellence, Kessels Road, Brisbane, QLD 4111, \\ Australia \\ * Senior Member, IEEE
}

Received 1 Nov 2016, revised 25 Nov 2016, accepted 30 Nov 2016, published 5 Dec 2016, current version 15 Dec 2016. (Dates will be inserted by IEEE; "published" is the date the accepted preprint is posted on IEEE Xplore ${ }^{\oplus}$; "current version" is the date the typeset version is posted on Xplore ${ }^{\circledR}$ ).

\begin{abstract}
Athlete movement data is integral for optimizing athlete-performance and can lead to reduced fatigue and in turn can mitigate injury risk. There is a substantial amount of scientific literature which investigates the ability of computervision and inertial sensor technologies to classify sport-specific movements. The coupling of automatic sport action labelling and athlete-monitoring data can significantly enhance athlete work-load monitoring. Two recent systematic reviews of the literature, pertinent to sport-specific movement classification, revealed that the majority of journal articles use athlete-dependent classification model training and evaluation methods. These methods can significantly enhance model classification performance, particularly with movements which have high inter-athlete technique variation. This is because it enables models to learn features distinctive to all athletes during training. This manuscript details the training and evaluation of supervised machine learning models to automatically classify running surface (athletics track, hard sand and soft sand) using features extracted from an upper-back inertial measurement unit sensor. Possible classification performance enhancement is demonstrated by comparing athlete independent and athlete dependent supervised machine learning methods. Using athlete dependent methods significantly increased the classification performance in terms of weighted average precision, recall, F1-score and accuracy $(p<0.05)$.
\end{abstract}

\section{Index Terms- Machine Learning, Inertial Sensors, Athlete-monitoring, Classification.}

\section{INTRODUCTION}

Supervised machine learning algorithms are powerful statistical tools which can be used for both classification and regression modelling. The underlying mathematics enables optimization of a range of different model-types via mapping input features to output target variables in a training data set. The optimized models can then perform class and value predictions on unseen data.

Sport is becoming increasingly data driven and as a result, supervised machine learning and artificial neural network models have been adopted for athlete monitoring tasks such as automatic sport-specific movement classification to enhance work-load quantification [1]-[6] and athlete-state prediction to identify when athletes are, for example, entering a fatigued state [7], [8].

When using supervised machine learning, the methodology used to divide data into training and validation sets is of upmost importance [9], particularly in athlete-monitoring data as 'one size doesn't fit all' [10]-[12].

The advent of microelectromechanical systems (MEMS) has facilitated the use of wearable inertial measurement unit sensors (IMUs) for performance monitoring in elite sport [13], [14]. As of 2018, two extensive systematic literature reviews explored the technologies, data collection procedures, feature engineering, validation methods and overall performance of machine learning models implemented in sport-specific movement classification tasks [1], [2]. Cust et al. [1] reviewed a total of 52 manuscripts and included both IMUs and vison-based classification algorithms. McGrath et al. [2] reviewed a total of 20 manuscript, however, only included records reporting IMUs classification algorithms. In [1] 15/52 (29\%) papers used a leave-one (or more)-subject-out (LOSO) model validation, while $22 / 52(42 \%)$ trained and tested on non-independent data (crossvalidation or train/test split) and 15/52 (29\%) did not clarify a validation method. In [2] 7/20 (35\%) papers used a LOSO validation method, 8/20 (40\%) evaluated on non-independent data (crossvalidation or train/test split) and 5/20 (25\%) did not clarify a validation method. In both systematic reviews, the LOSO validation method was the least used. In sport classification, unless new athlete data can immediately be added to the training data for new model creation, evaluating models on non-independent data can generate misleading classification performance. Consequently, the models are able to learn features specific to all the athletes. If an athlete independent to the trained models was tested, then the accuracy of the models would decrease significantly.

This paper documents the training and performance evaluation of six supervised machine learning models when classifying different running surfaces using features extracted from a IMUs located on an athlete's upper back. The models were trained and tested using both 
athlete-independent and athlete-dependent methodologies and the performance of each method is compared. While it is common knowledge that the athlete-dependent method will result in seemingly enhanced model classification ability (generalizability significantly reduced), to the best of the author's knowledge, a sport-specific case study demonstrating this is yet to be published. The conclusions of this paper will be of upmost importance when sporting organizations deliberate the training, selection and deployment of athlete sportspecific movement classification algorithms.

\section{METHODS}

\section{A. Participants}

Seven able-bodied participants $(4 \mathrm{M}, 3 \mathrm{~F})$ with a mean age $(32.4 \pm$ $17.89 \mathrm{yrs})$, mean height $(171.9 \pm 8.91 \mathrm{~cm})$ and mean weight $(70.3 \pm 16.87 \mathrm{~kg})$ consented to participate in the study (ethics approval number: GU 2017/587). The participants are from a diverse background of fitness and running experience (range of 9 hours training/week) and thus are a good representation of the general public.

\section{B. Experimental Design}

The participants were instructed to run $400 \mathrm{~m}$ at a light-moderate pace on soft, dry-sand. The participants then ran back at a similar pace on hard (water saturated) sand. This procedure was repeated at the on a 400m synthetic tartan surfaced running track. The participants were fitted with a single IMU, tightly secured in close proximity to the third thoracic vertebrae (T3) using a specially designed sports harness. The sensors axes orientation is illustrated in Figure 1.

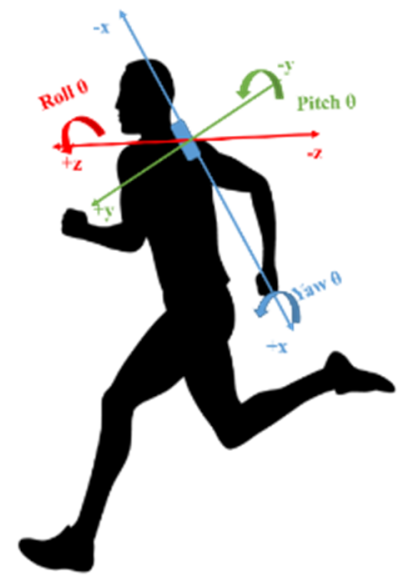

Figure 1. Sensor axes orientation in reference to the participant. $x$ is the superior-inferior axis, $y$ is the medial-lateral axis and $z$ is the anterior-posterior axis. Roll describes the angle about the anteriorposterior axis, pitch describes the angle about the medial-lateral axis and yaw describes the angle about the inferior-superior axis.

\section{IMU Sensor Technology}

An in-house 9DOF IMUs (SABELSense, Griffith University, Nathan, Australia - [15], [16] with $250 \mathrm{~Hz}$ sampling frequency, $\pm 16 \mathrm{~g}$ accelerometer, $\pm 2000 \mathrm{deg} / \mathrm{s}$ gyroscope, \pm 7 Gauss magnetometer and 23 gram weight was used in this study. The IMU was calibrated [17] before the trial and the data was logged locally to a memory card
(4GB micro SD card). The sensors 3-D orientation in terms of Euler's angles (roll, pitch and yaw) was recorded using Madgwick's AHRS algorithm (static root mean square (RMS) error $<0.8^{\circ}$, dynamic RMS error $<1.7^{\circ}$ ) [18].

\section{Algorithm}

\section{A. Feature Extraction}

When running on the $400 \mathrm{~m}$ athletics track, the Euler angle orientation begins to drift due to the participant moving in a circular radius. The feature extraction method used was similar to that reported by Dixon et al. [6]. Using MATLAB (MathWorks, Natick, MA, USA - https://www.mathworks.com/), a sliding window, 4 seconds duration with a 0.5 second slide between windows was used for feature extraction (capturing approximately 10-11 strides on each surface). This time duration is large enough to capture strides while eliminating drift due to the changes in heading direction. The Euler angle data windows are zeroed and made absolute to combat this heading-drift effect. If the data is still very spurious, then it is unlikely to make a significant contribution to the classification feature. The window was moved across 11-channels of the IMUs; Ax, Ay, Az, Amag, Gx, Gy, Gz, Gmag and, roll, pitch and yaw. Statistical features in both the time and frequency domains are extracted from each data window for each signal (mean, standard deviation, skewness, kurtosis, main frequency component etc.). The extracted features are shown in Table A1 of [19].

\section{B. Training/Validation Segmentation of Feature Data}

Two methods to divide the feature data into training and validation sets were used. Method 1 uses an athlete independent leave-onesubject-out train/test split; one subject is selected at random to evaluate model performance while the models are trained on six of the athletes. Method 2 uses an athlete-dependent train/test split, dividing the data by $75 / 25 \%$ (75\% for model training/25\% for model testing). The two methods were used to demonstrate the impact of individual features for each participant on model classification performance. Method 1 resulted in 1537, 1237 and 944, while method 2 resulted in 1720, 1390 and 1140 observations of soft sand, hard sand and athletics track running surfaces respectively being used for model training. This left 183, 153 and 196, and 413, 341 and 309 observations of soft sand, hard sand and athletics track running surfaces for model evaluation using methods 1 and 2 respectively.

\section{Feature Engineering}

All feature engineering and model training was conducted in Python (Python Software Foundation, https://www.python.org/) using the scikit-learn and pandas modules [20], [21]. Each feature is standardized (normalized to a range of 0-1) using the mean and standard deviation of the training data. Principal component analysis (PCA) [22] was used for feature dimensionality reduction (FDR). PCA transforms features into orthogonal variables (principal components) which each represent a percentage of variance within the feature space. Only those principal components required to explain 95\% variance within the feature space were retained in the training data (PCA reduced feature dimensionality from 132 features to 45). PCA removes noisy and correlated features and consequently reduces 
computational expense, model training time and overfitting. PCA is an unsupervised FDR technique and therefore does not retain/eliminate features which are only important/redundant for a specific athlete. This was thought to be advantageous for the comparison of the athlete-independent and athlete-dependent methods. A supervised FDR technique such as linear discriminant analysis (LDA) may result in amplified model overfitting, which presents bias towards the athlete-dependent method. Also PCA has been shown to outperform LDA when the number of training samples per class is small, as is the case in this investigation [23].

\section{Model Training and Evaluation}

Six different types of machine learning models were trained and evaluated. These models have proven effective classifying sportspecific movements using inertial sensor data [1], [2], [20]. The trained models are logistic regression ( $\mathrm{LR}[\mathrm{C}=0$, penalty $=12$, solver $=$ lbfgs)), support vector machine (SVM $[\mathrm{C}=1$, gamma $=$ scale $]$ ) with linear (LSVM) and Gaussian rbf (GSVM) kernels, multi-layered perceptron neural network (MLP-NN [activation $=$ relu, alpha $=$ 0.0001 , hidden layer sizes $=8,8,8\{3$ hidden layers with 8 nodes each $\}$, learning rate $=$ constant, solver $=$ adam $]$, random forest $(\mathrm{RF}$ [criterion = gini, maximum features $=\sqrt{\text { number of features }}$, number of estimations $=20]$ ) and gradient boosting (XGB [criterion = friedman_mse, loss $=$ deviance, $\max$ depth $=3$, maximum features $=$ none, number of estimators $=100]$ ) (no hyperparameter tuning). The models were evaluated using the weighted average of statistical metrics; precision, recall, F1-score and overall classification accuracy. The performance of the models using athlete-dependent and independent training/validation segmentation methods was compared using a paired t-test $(\alpha=0.05)$.

\section{RESULTS}

Using the train/test split method for model training and evaluation resulted in statistically significant increases in the weighted average precision, recall, F1-score and accuracy metrics ( $p=0.0002, p=$ $0.0004, p=0.0004, p=0.0004$ respectively) across all model-types. An estimation plot highlighting this significant difference for model F1-score is shown in Figure 2.

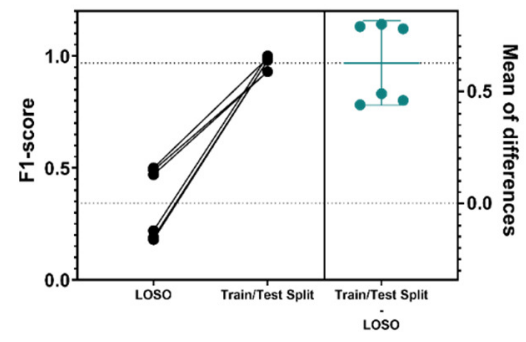

Figure 2. Estimation plot demonstrating the difference in model weighted average $\mathrm{F} 1$-score using LOSO and train/test split, training and validation methods.

Using a train/test split model training and validation method, which exposes models to individual features respective to all the participants, results in a significant increase in model predictive ability. The respective evaluation metrics for each validation method and all model types are shown in Figure 3 (a-d).

When each model can learn features respective to a participant's running style on each surface type, the classification performance is enhanced significantly. If features extracted from a T3 inertial sensor were to be used for generalized surface classification purposes, then the participant pool would need to be increased dramatically. The best performing models using the LOSO and train/test split validation methods were MLP-NN and GSVM respectively. The classification ability of these two models can be expressed by confusion matrices (Figures 4 and 5).

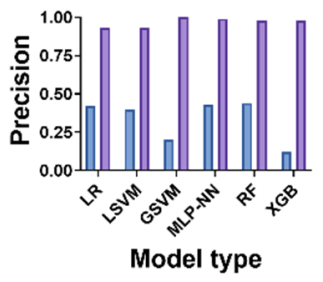

(a)

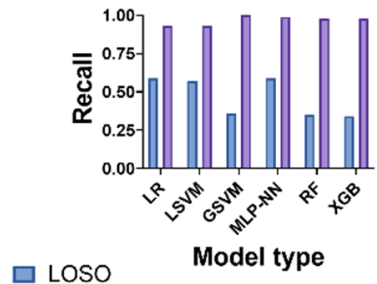

(b) $\square$ Train/Test Split

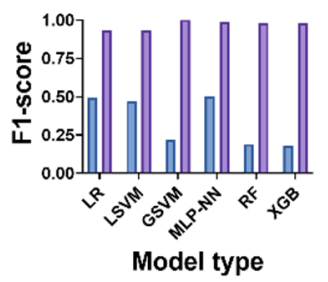

(c)

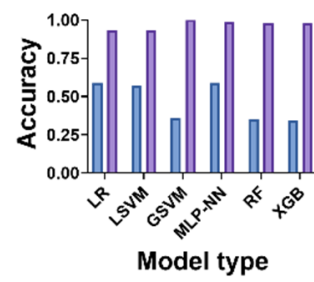

(d)
Figure 3. Weighted average for each validation method and model type; (a) precision score, (b) recall score, (c) F1-score, and (d) accuracy score.

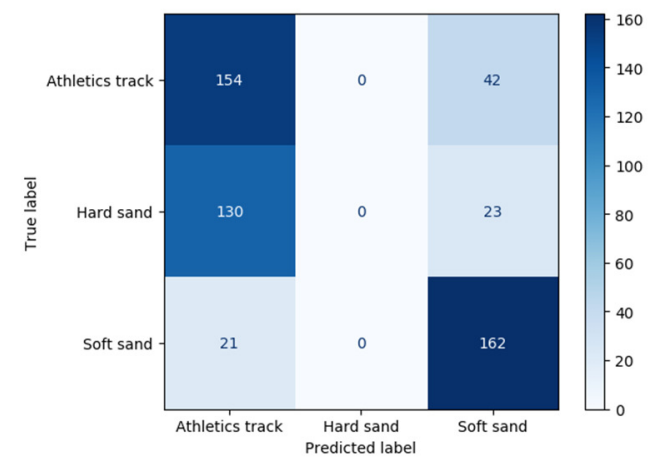

Figure 4. Confusion matrix demonstrating classification performance for MLP-NN using LOSO training and validation.

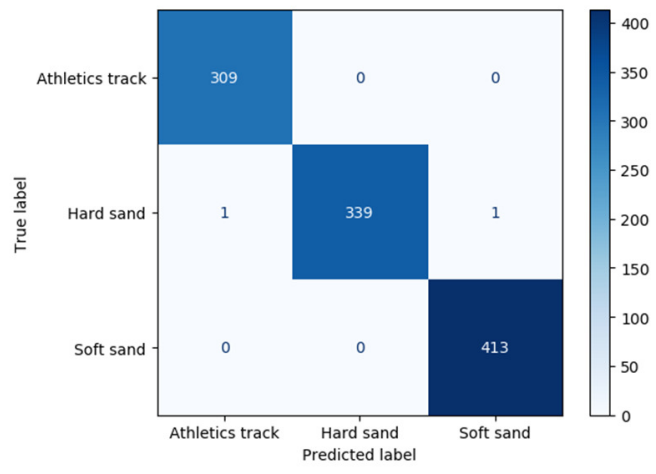

Figure 5. Confusion matrix demonstrating classification performance for GSVM using 75/25 train/test split training and validation method. 
Figure 4 shows that the MLP-NN was able to differentiate a soft sand running surface from hard sand and the athletic track with moderate success even using the LOSO method; soft sand precision, recall and F1-score was $0.71,0.89$ and 0.79 respectively. This is not surprising considering the visual difference in gait when running on soft sand compared to the other two harder surfaces. Hard sand and the athletic track are more similar in surface properties and so it was anticipated that misclassifications would occur more frequently for these surface types. Using a train/test split training and validation method, the GSVM model performed exceptionally well (overall accuracy $=0.99^{\prime}$ ) and there was only 1 misclassification between the athletics track and hard sand classes ( 2 misclassifications in total) (Figure 5). There is significant benefit in including data from all athletes when implementing automated athlete-load monitoring and sport-specific movement classification algorithms.

\section{DISCUSSION}

This case study demonstrates the enhanced model classification performance using athlete-dependent train/test split evaluation methods $(\mathrm{p}<0.05)$. In athlete-monitoring, the way in which individuals perform certain movements can be quite unique and allowing models to learn these distinct features clearly increases classification performance. To create a generalized sport-action classification model which has high performance on completely independent athletes requires training data from a large pool of athletes with varying technique, physical properties (height, mass etc.), skill level and fitness level. For privacy reasons, access to athlete performance data is limited and as a result, complete athleteindependent sport-action classification models can be hard to build, particularly when the action can vary significantly from person to person (e.g. running style on different surfaces). For this reason, the authors recommend that sporting organizations looking to add automatic tagging of sporting actions to enhance athlete-load monitoring should include the features of all their athletes in a classification model. When a new athlete joins the organizations, a calibration session is recommended, allowing the classification model to learn features unique to the new athlete. Automated running surface classification using an upper-back located sensor, albeit being athletedependent, is also of significant value for the purpose of accurately modifying athlete session work-rate estimation values when direct physiological sensors (e.g. heart rate monitors) are unavailable.

\section{ACKNOWLEDGMENT}

This project was aided by an affiliation of the lead researcher with the Sport Performance Innovation and Knowledge Excellence (SPIKE) unit of the Queensland Academy of Sport. M.T.O.W. received a $\mathrm{PhD}$ scholarship funding from Griffith University.

\section{REFERENCES}

[1] E. E. Cust, A. J. Sweeting, K. Ball, and S. Robertson, "Machine and deep learning for sport-specific movement recognition: a systematic review of model development and performance," J. Sports Sci., vol. 37, no. 5, pp. 568-600, Mar. 2019, doi: 10.1080/02640414.2018.1521769.

[2] J. McGrath, J. Neville, T. Stewart, and J. Cronin, "Upper body activity classification using an inertial measurement unit in court and field-based sports: A systematic review," Proc. Inst. Mech. Eng. Part P J. Sports Eng. Technol., vol. 0, no. 0, p. 1754337120959754, 2020, doi: 10.1177/1754337120959754.

[3] O. Steven Eyobu and D. Han, "Feature representation and data augmentation for human activity classification based on wearable IMU sensor data using a deep LSTM neural network," Sensors, vol. 18, no. 9, pp. 1-36, Aug. 2018, doi: $10.3390 / \mathrm{s} 18092892$.

[4] S. Wan, L. Qi, X. Xu, C. Tong, and Z. Gu, "Deep learning models for real-time human activity recognition with smartphones," Mob. Netw. Appl., vol. 25, no. 2, pp. 743-755, Apr. 2020, doi: 10.1007/s11036-019-01445-x.

[5] Z. Gao, H.-Z. Xuan, H. Zhang, S. Wan, and K.-K. R. Choo, "Adaptive fusion and category-level dictionary learning model for multiview human action recognition," IEEE Internet Things J., vol. 6, no. 6, pp. 9280-9293, Dec. 2019, doi: 10.1109/JIOT.2019.2911669.

[6] P. C. Dixon et al., "Machine learning algorithms can classify outdoor terrain types during running using accelerometry data," Gait Posture, vol. 74, pp. 176-181, Oct. 2019, doi: 10.1016/j.gaitpost.2019.09.005.

[7] G. A. Einicke, H. A. Sabti, D. V. Thiel, and M. Fernandez, "Maximum-entropy-rate selection of features for classifying changes in knee and ankle dynamics during running," IEEE J. Biomed. Health Inform., vol. 22, no. 4, pp. 1097-1103, Jul. 2018, doi: 10.1109/JBHI.2017.2711487.

[8] C. Buckley et al., "Binary classification of running fatigue using a single inertial measurement unit," in 2017 IEEE 14th International Conference on Wearable and Implantable Body Sensor Networks (BSN), Eindhoven, Netherlands, May 2017, pp. 197-201, doi: 10.1109/BSN.2017.7936040.

[9] A. Koul, C. Becchio, and A. Cavallo, "Cross-validation approaches for replicability in psychology," Front. Psychol., vol. 9, Jul. 2018, doi: 10.3389/fpsyg.2018.01117.

[10] C. E. Diss, "The reliability of kinetic and kinematic variables used to analyse normal running gait," Gait Posture, vol. 14, no. 2, pp. 98-103, Oct. 2001, doi: 10.1016/S0966-6362(01)00125-4.

[11] ChewYean Yam, M. S. Nixon, and J. N. Carter, "On the relationship of human walking and running: automatic person identification by gait," in Object recognition supported by user interaction for service robots, Quebec City, Que., Canada, 2002, vol. 1, pp. 287-290, doi: 10.1109/ICPR.2002.1044691.

[12] S. Khandelwal and N. Wickström, "Evaluation of the performance of accelerometerbased gait event detection algorithms in different real-world scenarios using the MAREA gait database," Gait Posture, vol. 51, pp. 84-90, Jan. 2017, doi: 10.1016/j.gaitpost.2016.09.023.

[13] H. G. Espinosa, J. B. Shepherd, D. V. Thiel, and M. T. O. Worsey, "Anytime, anywhere! Inertial sensors monitor sports performance," IEEE Potentials, vol. 38, no. 3, pp. 11-16, May 2019, doi: 10.1109/MPOT.2019.2896343.

[14] V. Camomilla, E. Bergamini, S. Fantozzi, and G. Vannozzi, "Trends supporting the in-field use of wearable inertial sensors for sport performance evaluation: A systematic review," Sensors, vol. 18, no. 3, p. 873, Mar. 2018, doi: 10.3390/s 18030873 .

[15] J. B. Shepherd, D. V. Thiel, and H. G. Espinosa, "Evaluating the use of inertialmagnetic sensors to assess fatigue in boxing during intensive training," IEEE Sensors. Lett., vol. 1, no. 2, pp. 1-4, 2017, doi: 10.1109/LSENS.2017.2689919.

[16] D. V. Thiel, J. B. Shepherd, H. G. Espinosa, M. Kenny, K. Fischer, M. T. O. Worsey, A. Matsuo, and T. Wada, "Predicting ground reaction forces in sprint running using a shank mounted inertial measurement unit," MDPI Proc., vol. 2, no. 6, 2020, doi: 10.3390/proceedings2060199.

[17] A. Lai, D. A. James, J. P. Hayes, and E. C. Harvey, "Semi-automatic calibration technique using six inertial frames of reference," in Proceedings, Perth, Australia Mar. 2004, vol. 5274, pp. 1-13, doi: 10.1117/12.530199.

[18] S. O. H. Madgwick, A. J. L. Harrison, and R. Vaidyanathan, "Estimation of IMU and MARG orientation using a gradient descent algorithm," in 2011 IEEE International Conference on Rehabilitation Robotics, Zurich, Jun. 2011, pp. 1-7, doi: 10.1109/ICORR.2011.5975346.

[19] M. T. O. Worsey, H. G. Espinosa, J. B. Shepherd, and D. V. Thiel, "An evaluation of wearable inertial sensor configuration and supervised machine learning models for automatic punch classification in boxing," IoT, vol. 1, no. 2, pp. 360-381, Nov. 2020, doi: 10.3390/iot1020021.

[20] F. Pedregosa et al., "Scikit-learn: Machine learning in python," J. Mach. Learn. Res., vol. 12, pp. 2825-2830, 2011

[21] W. McKinney, "Data structures for statistical computing in python," Austin, Texas, 2010, pp. 56-61, doi: 10.25080/Majora-92bf1922-00a

[22] H. Abdi and L. J. Williams, "Principal component analysis," Wiley Interdiscip. Rev. Comput. Stat., vol. 2, no. 4, pp. 433-459, Jul. 2010, doi: 10.1002/wics.101.

[23] A. M. Martinez and A. C. Kak, "PCA versus LDA," IEEE Trans. Pattern Anal. Mach. Intell., vol. 23, no. 2, pp. 228-233, Feb. 2001, doi: 10.1109/34.908974. 